\title{
Characterization of Electrowetting Processes through Force Measurements
}

Nathan B. Crane

Brigham Young University - Provo, nbcrane@byu.edu

Pradeep Mishra

University of South Florida

Alex A. Volinsky

University of South Florida

Follow this and additional works at: https://scholarsarchive.byu.edu/facpub

Part of the Other Mechanical Engineering Commons

\section{Original Publication Citation}

N. B. Crane, P. Mishra, and A. Volinsky, "Characterization of Electrowetting Processes through Force Measurements," Review of Scientific Instruments, Vol 81, 2010, 043902.

\section{BYU ScholarsArchive Citation}

Crane, Nathan B.; Mishra, Pradeep; and Volinsky, Alex A., "Characterization of Electrowetting Processes through Force Measurements" (2010). Faculty Publications. 5361.

https://scholarsarchive.byu.edu/facpub/5361 


\title{
Characterization of Electrowetting Processes through Force Measurements
}

\author{
Nathan B. Crane, Pradeep Mishra, Alex A. Volinsky \\ Department of Mechanical Engineering \\ University of South Florida \\ Tampa, FL 33620
}

\section{ABSTRACT}

A new method of characterizing electrowetting is presented in which the forces applied to a modified nanoindenter tip by a test water droplet are measured. A droplet is trapped between the flat nanoindenter tip and the test substrate containing the necessary electrodes. When voltage is applied to the electrodes in the substrate, lateral and normal forces are exerted on the tip and measured by the nanoindenter transducer. Proper selection of the tip geometry permits direct prediction of the resulting in-plane lateral forces using analytical formulas derived from the Young-Lippmann equation. Experimental results show good agreement with both analytical and numerical predictions. Numerical modeling using Surface Evolver shows that the lateral forces are relatively insensitive to most alignment errors. The analytical model is most accurate for small tip/substrate gaps. Evaporation of the test liquid can introduce modest errors in long measurements, but compensation methods are presented. The nanoindenter sensor provides microNewton force resolution with fast response time. As the droplet undergoes almost no movement, the fluid dynamics have minimal impact on the measured forces and transient electrowetting events are readily detected. Experimental results show significant response at frequencies up to $40 \mathrm{~Hz}$. This setup is useful in measuring electrowetting responses at high speeds and in measuring system degradation processes. 


\section{INTRODUCTION}

Electrowetting was first identified by Lippmann in 1857 , but recent advancements have led to an explosion of potential applications ${ }^{1}$. This effect has been demonstrated for a wide range of liquids, including water, common solvents, and ionic liquids ${ }^{2,3}$. New applications that have been investigated include digital microfluidics ${ }^{4-6,7}$, responsive $\operatorname{cooling}^{8}$, focusing lenses ${ }^{9}, 10$, and flexible displays ${ }^{11}$. Typically, the electrowetting liquid is contacted by one electrode from above while a second insulated electrode is located beneath the droplet as shown in Figure 1. The insulating dielectric layer is commonly coated with a thin hydrophobic coating (not shown). The incorporation of a dielectric layer between the liquid and electrodes to reduce electrochemical reactions is critical to many of these applications. Variations include grounding the droplet from below and "floating drop" configurations 12 . These provide simplified electrical connections and control for some applications. However, the electrowetting response still involves complex coupling of electrical, chemical, fluid, and surface properties. As such, many aspects of electrowetting behavior are not yet fully understood. These include dielectric charging ${ }^{13}{ }^{14}$, asymmetric (polarity dependent) electrowetting responses ${ }^{15}$, and saturation phenomena ${ }^{16-18}$.

Electrowetting can be addressed from various perspectives (thermodynamic, electrochemical, energy minimization, electromechanical), but the electromechanical approach has been receiving increased attention $^{19}$. However, contact angle measurement remains the predominant experimental method for investigating electrowetting phenomena. As a first approximation, the dielectric layer can be treated as a parallel plate capacitor and the liquid as a conductor. For DC applied voltages, the interface voltage is not impacted by the liquid resistance. Under these assumptions, the contact angles $\left(\theta_{1}\right)$ when the voltage $(V)$ is applied between the substrate electrode and the droplet will depend on the liquid's contact angle 
$\left(\theta_{0}\right)$ without voltage applied. These can then be related to the thickness $(\delta)$ and dielectric constant $\left(\varepsilon_{0}\right.$, $\left.\varepsilon_{\mathrm{R}}\right)$ of the dielectric layer by the Young-Lippmann equation ${ }^{1}$

$$
\cos \theta_{1}=\cos \theta_{o}+\frac{\varepsilon_{o} \varepsilon_{r} V^{2}}{2 \gamma_{l v} \delta}
$$

The quantity $\varepsilon_{0} \varepsilon_{r} V^{2} / 2 \delta$ is simply the energy stored per unit area in a parallel plate capacitor so that the change in apparent energy of the droplet/substrate interface is equal to the capacitive energy storage. This relationship generally agrees with the test data below a critical voltage referred to as the saturation voltage.

High speed imaging permits high time resolution of contact angle data, but when the interface is in motion, the contact angle depends on both the fluid dynamics and electrowetting phenomena. However, since the Young-Lippmann equation is based on equilibrium conditions, the electrowetting behavior cannot be directly measured under changing voltages. Verheijen and Prins ${ }^{20}$ developed an alternative approach to electrowetting characterization in which the capacitance between the drop and the substrate is measured. As the contact angle decreases, the droplet spreads-increasing the effective area of the capacitor. The resulting capacitance change can be correlated with the contact angle to provide highly repeatable measurements. However, during rapid changes, the measurement still depends on both fluid dynamics and electrowetting effects. Recent work has shown that dynamic effects significantly affect the shape of mm-scale droplets ${ }^{21}$ and even the saturation voltages ${ }^{17}$ over a large range of voltage frequencies.

Alternatively, the electrowetting response can be measured in terms of a force. If the droplet is constrained from moving to an equilibrium position, a force will be applied to the constraint. This force can be measured and related to the electrowetting response. This work reports on a force-based electrowetting measurement system in which the droplet is trapped between two plates, at least one of 
which is wetted by the fluid. The geometrical constraints on the droplet minimize the fluid motion during testing so that electrowetting changes are separated from fluid dynamics effects. Additionally, the electrowetting actuation forces are of more direct interest than contact angle in many electrowetting applications - particularly where electrowetting is used to move a droplet or something wetted by the droplet $^{22,23}$. By restraining movement of the three phases contact line, this approach may provide new insights into electrowetting saturation ${ }^{24}$.

Models of electrowetting forces have been developed previously using techniques from continuum electromechanics ${ }^{19,} 25,26$ to atomistic simulations ${ }^{27}$. However, limited force measurements of electrowetting systems have been done. Some have focused on the performance of a specific device such as a microgripper ${ }^{23}$. The others are based on nanoscale measurements. For example, Guan et al. used an AFM to measure electrowetting forces of water condensate on PMMA as a function of applied voltage $^{28}$, and Chen et al. measured the force of mercury wetting a carbon nanotube ${ }^{29}$. These tests provide valuable information about nanoscale phenomena, but the results are difficult to extrapolate to microscale and mesoscale situations where most electrowetting applications are studied. Electrowetting force measurements at a larger scale provide a valuable means of assessing predictive force models, characterizing the dielectric layers, tracking their degradation over time, studying phenomena such as trapped charges, detecting electrochemical corrosion, and studying electrowetting response on textured surfaces. A measurement method that addresses these needs is described below. Numerical models are used to evaluate the sensitivity of this measurement system to key measurement parameters. Experimental measurements from the fore measurement system are reported for steady and dynamic inputs. 


\section{Electrowetting Force Measurement Apparatus}

The electrowetting force (EWF) is measured using a modified Hysitron Triboindenter nanoindentation apparatus ${ }^{30}$. The commercial nanoindenter has microNewton force resolution in two axes (normal and lateral). Figure 2(a) illustrates how it can be adapted for EWF measurement by attaching a flat $9 \mathrm{~mm} \times 9 \mathrm{~mm} \times 0.15 \mathrm{~mm}$ glass plate to a custom tip so that it is parallel to a test substrate. The test substrate is patterned to contain two or more electrodes covered with a dielectric and hydrophobic top surface. Tests are performed by placing a droplet on the substrate and lowering the glass plate until it is entirely wet with the test solution. The droplet should be positioned so that it bridges the gap between the electrodes. The glass surface was chosen for testing aqueous solutions because in general, they wet the glass well. The test substrate contains one or more electrodes covered by a dielectric layer as illustrated in Figure 2(a). Once the droplet is sandwiched between the substrate and the glass plate, it will move with the glass plate. For optimal alignment of the glass plate to the substrate, the glass plate can be attached to the indenter by adhesive using the substrate as an alignment guide. The nanoindenter has calibrated optical alignment system that permits positioning of the plate center to within $\sim 1 \mu \mathrm{m}$ and alignment of the plate edges with the electrode edges to within $0.25^{\circ}$.

While many electrical configurations of the substrate are possible, two of primary interest are shown in Figure 2(c). The electrowetting force model can be developed using the lumped parameter electrical model illustrated in the figure. As the gap between the glass plate and the substrate decreases, the contact region of the test solution on the substrate approaches the area and shape of the glass plate as seen in Figure 2(b). The droplet forms a capacitor with the electrodes under the dielectric. Under this condition, the area of each capacitor can be calculated as a function of the offset $(y)$ of the plate's center from the electrode boundary. Given the gap $(g)$ between adjacent electrodes and the length of the glass plate $(L)$, the left and the right areas $\left(A_{L}, A_{R}\right)$ are given by 


$$
A_{L}=L(L-g-2 y) / 2, A_{R}=L(L-g+2 y) / 2, \text { for }-(L-g) / 2<y<(L-g) / 2
$$

Modeling the droplet interfaces as two parallel plate capacitors with capacitance $\left(C_{L}, C_{R}\right)$, the total capacitive energy $\left(E_{c a p}\right)$ is given by

$$
\begin{gathered}
E_{\text {cap }}=\left(C_{L} V_{L}^{2}+C_{R} V_{R}^{2}\right) / 2=\varepsilon_{0} \varepsilon_{r}\left(A_{L} V_{L}^{2}+A_{R} V_{R}^{2}\right) / 2 \delta \\
V_{L}=V_{\text {tot }} A_{R} /\left(A_{L}+A_{R}\right), V_{R}=V_{\text {tot }} A_{L} /\left(A_{L}+A_{R}\right),
\end{gathered}
$$

where $V_{L}$ and $V_{R}$ are the voltages on the left and right sides, respectively. The lateral force $\left(F_{y}\right)$ in the system can be found by combining equations (1)-(3) and differentiating the energy with respect to the displacement so that

$$
\begin{gathered}
E=\frac{\varepsilon_{0} \varepsilon_{r} L V_{t o t}^{2}}{2 \delta}\left(\frac{L-g}{4}-\frac{y^{2}}{L-g}\right) \\
F_{y}=d E / d y=-\frac{\varepsilon_{0} \varepsilon_{r} L V_{t o t}^{2}}{(L-g) \delta} y
\end{gathered}
$$

In the grounded droplet configuration, the lumped parameter model consists of a single capacitor so that the voltage at the interface is the total voltage applied. The force is then

$$
F_{y}=-\varepsilon_{0} \varepsilon_{r} L V_{t o t}^{2} / \delta
$$

Note that while the contact angle depends on the liquid-vapor surface energy $\left(\gamma_{l v}\right)$, the predicted force at a particular voltage is independent of the surface energies as long as the Young-Lippmann equation holds true $\left(V_{\text {tot }}<V_{\text {sat }}\right)^{1,16}$. Actuation liquids with higher saturation voltages will produce higher peak forces, but at low voltages, the electrowetting force is predicted to be independent of the electrowetting liquid used. Exceptions have been observed as seen in Figure 10(a). These are believed to be due to material and dynamic effects such as ion transport into polymer dielectric layers through diffusion and/or defects that are not included in this model. 
The above model assumes that the liquid interface with the substrate matches both the shape and location of the glass plate. The validity of this assumption was evaluated by modeling the floating droplet configuration using Version 2.26 of Surface Evolver ${ }^{31}$. For this analysis, gravity and surface tension contributions were included. The equilibrium surface is found by a gradient-based solution of the shape that minimizes the total energy. In the model (Figure 3), the interfacial energy is calculated using the Young-Lipmann equation based on the contact area of the fluid over each electrode. Similar

methods were used by Lienemann to simulate droplet motion by electrowetting ${ }^{32}$. The forces applied to the top plate are calculated numerically from the derivative of the system energy with respect to the displacement of the plate in the direction of interest $\left(F_{j}=d E / d u_{j}\right.$, where $u_{j}$ is a unit vector in the direction of interest).

The test configuration was modeled with a $9 \mathrm{~mm} \times 9 \mathrm{~mm}$ plate. Other parameters are summarized in Table 1. Figure 4 shows that the Surface Evolver models closely match the results predicted by Equation (5) for the floating droplet case. As the gap increases, the force-y-offset relationship remains linear, but the slope decreases. This is due to inaccuracies in the assumption that the droplet shape matches the plate shape.

\section{Force Sensitivity to Alignment Accuracy}

Equations (1)-(5) were based on the alignment of the indenter plate parallel to the substrate and a "small" gap between the plate and the substrate. However, practical implementation of these methods requires an assessment of the sensitivity of the measurements to the alignment accuracy ${ }^{33}$. Rotations were applied to the plate in the Surface Evolver models using the coordinate system shown in Figure 3 and the force was calculated as a function of rotation for a $3 \mathrm{~mm}$ offset $(y=3 \mathrm{~mm})$. The results are summarized in Figure 5. The fluid volume $\left(V_{f}\right)$ is set to the projected volume below the perfectly aligned plate $\left(V_{f}=h L^{2}\right)$. The analysis was started with the minimum angle error $\left(-5^{\circ}\right)$. The plate was 
rotated in $0.25^{\circ}$ increments to the maximum error and then reduced back to the minimum error. The force difference between increasing and decreasing angles is a function of the convergence accuracy of the Surface Evolver models, which are seen to be below $1 \%$ of the total force.

Normal forces show large sensitivity to alignment errors in all axes except the z-axis, thus the normal force data provides little useful information. However, the lateral forces are relatively insensitive to angular misalignments of all types. Rotations around the y-axis and z-axis introduce force errors of approximately $1 \%$ at $5^{\circ}$. The force errors are much more significant for $\mathrm{x}$-axis rotations. Positive rotational errors (thinner glass/substrate gaps on the small area, high voltage side) produced larger errors $\left(4.4 \%\right.$ at $\left.+5^{\circ}\right)$ than negative rotations $\left(1.9 \%\right.$ at $\left.-5^{\circ}\right)$. $X$ - and $y$-axis alignment errors can be maintained well below five degrees by using the substrate as an alignment aid. Similar alignment accuracy in the zaxis can be achieved by optically verifying the alignment of the glass plate to the machine axes before bonding.

As seen in Figure 6, the force magnitude increases as the plate to substrate gap $(h / L)$ decreases. However, the volume of the liquid is also of importance. While the initial analysis assumes that the liquid volume is the same as the projected volume, this may be difficult to achieve in practice due to the accuracy of liquid dispensing and evaporation changing the liquid volume over time. The impact of variable liquid volume was analyzed using the Surface Evolver models at three different values of the gap ratio $(h / L)$. For each gap ratio, the volume ratio $\left(V_{f} / h L^{2}\right)$ was varied was decreased from 1.0 to 0.85 , increased to 1.05 , and then decreased back to 1.0 to evaluate hysteresis and model convergence. The results are summarized in Figure 7.

As in the previous case, the lateral forces are much less sensitive to variations in volume than the normal forces. The lateral forces decrease as much as $6 \%$ from their peak magnitude as the volume ratio decreases to 0.85 (15\% decrease). The largest lateral forces are seen for a volume ratio at or slightly 
below unity. This data suggests that testing should be performed at volume ratios near unity. Of note, the large gap ratios (variation $<5 \%$ for $\mathrm{h} / \mathrm{L}=0.056$ ) are less sensitive to the evaporation effects than small gap ratios (variation $\sim 10 \%$ for $h / L=0.017$ ). As the volume of many test liquids will decrease during testing due to evaporation, large gap ratios are preferred for their decreased sensitivity to the declining liquid volume. While this large height ratio slightly decreases lateral force (Figure 4), the decreased volume sensitivity is a significant advantage. For $h / L=0.017$, there is a significant hysteresis in the normal forces for volume ratios above 1.0 due to the formation of a large protrusion of liquid at high volume ratios. However, despite this large change in shape, the lateral forces are very stable.

During longer tests, the drift in the lateral forces due to evaporation may prevent accurate detection of the electrowetting behavior changes. This could be addressed by using a saturated atmosphere or submerging the glass plate in a second immiscible liquid to reduce the evaporation rate. Similar

arrangements have been made previously for indentation in liquids ${ }^{34}$. Alternatively, the gap height could be decreased at a steady rate to maintain a constant volume ratio as the gap decreases. Figure 8 shows that this dramatically reduces the variation in the lateral forces with changing liquid volume. A $25 \%$ reduction in liquid volume resulted in just $1 \%$ change in the lateral force when the volume ratio was maintained constant. As the normal forces are zero at a volume ratio of unity, the volume ratio could be maintained at unity by minimizing the magnitude of the normal forces during testing.

\section{Experimental Data}

Test structures were prepared on silicon wafers with a $500 \mathrm{~nm}$ layer of thermal oxide. A $300 \mathrm{~nm}$ thick aluminum film was deposited by sputtering and patterned photolithographically via a wet etching technique. CYTOP ${ }^{\text {TM }}$ 809M, a fluoropolymer produced by Asahi Glass company, was spin coated over the patterned wafer in two layers. After the first coating of CYTOPTM wafers were baked at $90{ }^{\circ} \mathrm{C}$ for 30 minutes. Then a second layer of CYTOPтM was spin coated and final baking was done at $150{ }^{\circ} \mathrm{C}$ for 1 
hour. The thickness of the CYTOPTM films was measured by a step profilometer with typical thickness of the two CYTOPтM layer ranging from 2.0 to $2.2 \mu \mathrm{m}$. Electrical connections to the aluminum electrodes were made using conductive copper tape.

Prepared substrates were mounted on the Hysitron Triboindenter test platform. A $55 \mu 1$ droplet of a $1 \mathrm{mM} \mathrm{Na}_{2} \mathrm{SO}_{4}$ solution was dispensed on the substrate with a micrometer syringe. Larger droplets were used than in the simulations to allow for drop evaporation during testing. The glass plate was then moved into the test position-typically $3 \mathrm{~mm}$ offset from the droplet center and lowered until the entire glass plate surface was wet with the salt solution. Voltage was then applied to the substrate electrodes while the force was measured using the Hysitron transducer. DC voltages were applied using a Matusada RG-360-0.2 power supply. Dynamic electrical inputs were amplified from a function generator using a Matsusada AMS-1B30 amplifier.

In a typical test, 50-150 V was applied across the electrodes. In a series of tests, increasing DC voltages were applied to the substrate electrodes while the resulting forces were recorded. From 50 to $110 \mathrm{~V}$, the maximum lateral force compares well with predictions from Eq. (5) and Surface Evolver models as seen in Figure 8. The measurement method is sensitive to defects in the dielectric layer. This is helpful in studying the dielectrics, but the current Cytop dielectric layers frequently contain local defects that introduce force deviations preventing accurate determination of the method repeatability. This peak force data gives information comparable to the contact angle data, but the time-response data provides clues to the nature of the degradation mechanisms. Figure 9(a) shows the force response data and applied voltage for the above test case versus time. The data show a fast response time $(<<1 \mathrm{~s})$ and a residual force after removal of the applied voltage. This residual force could be related to charge trapping in the dielectric layer. It also captures a sharp change in the applied force approximately one second after the application of $110 \mathrm{~V}$ (Figure 9(b)). After 1.2 seconds, the force suddenly drops from 
$349 \mu \mathrm{N}$ to $318 \mu \mathrm{N}$ in the 0.05 seconds interval between data points, after which a steady decline in force ensued. When $120 \mathrm{~V}$ was subsequently applied, the force was well below the predicted value (Figure 8). The sharp drop in force and subsequent change in behavior suggests a sudden local failure in the dielectric coating. Transient spikes have been seen with many test liquids and are associated with electrode corrosion. Depending on the electrolyte, voltage polarity, and dielectric layer quality, the forces may increase, decrease, or remain unchanged with prolonged voltage exposure. The high time resolution of this data provides opportunity for new insight into the electrowetting response and its degradation. Additional insight could be obtained by integrating EWF with current measurements similar to B. Raj et al. ${ }^{35}$.

The electrowetting force response at high frequencies was measured to evaluate the frequency response limits of the EWF method. Using the $9 \mathrm{~mm} \times 9 \mathrm{~mm}$ plate with a $55 \mu \mathrm{l}$ droplet, a $75 \mathrm{~V}$ square pulse was applied to the samples at varying frequencies. Figure 10(a) shows data from a $1 \mathrm{~Hz}$ pulse for three different liquids (DI water, $1 \mathrm{mM} \mathrm{NaCl}, 1 \mathrm{M} \mathrm{NaCl}$ ). The initial response to the step input is typical of a damped second order system with an overshoot and decaying oscillation. The oscillation frequency was estimated by measuring the distance between the first two peaks. Average frequencies were 41,52 , and $55 \mathrm{~Hz}$ for DI water, $1 \mathrm{mM} \mathrm{NaCl}$, and $1 \mathrm{M} \mathrm{NaCl}$, respectively. The frequency differences may be related to the variation in liquid volume ratios at the time of the test. Figure 10(b) summarizes the peak to peak amplitudes measured for each liquid as a function of frequency when subject to a $75 \mathrm{~V}$ square pulse. The high salt concentration $(1 \mathrm{M} \mathrm{NaCl})$ showed a decreased response above $4 \mathrm{~Hz}$, but the lower salt concentration fluids (DI water and $1 \mathrm{mM} \mathrm{NaCl}$ ) showed good response out to $40 \mathrm{~Hz}$. When the transducer was tapped with the plate attached, the first vibration mode was measured as $285 \mathrm{~Hz}$. This suggests that the transducer response is not limiting the EWF measurement. Further study is required to identify the parameters which limit the EWF frequency response in the test system. 


\section{Conclusions}

A method for measuring the force applied to a drop by the electrowetting effect has been presented. The method uses a custom flat tip attached to a commercial nanoindenter with a two-axis force transducer. This system provides a high resolution force measurement with a very fast response time. The EWF measurement method is shown to have low sensitivity of the lateral force to alignment and liquid volume errors. In contrast, the normal force is very sensitive to these errors making it very difficult to interpret normal force data. Thus, analysis focuses on the lateral force data. The geometry of the system permits straightforward estimates of the force from analytical predictions. These predictions show good agreement with numerical models and experimental data. As this method minimizes liquid motion, it permits close examination of the electrowetting phenomena during dynamic events. This method will be useful in detecting degradation in the electrowetting response over time and identifying the degradation mechanisms. It could also be used to identify mechanisms for increasing the speed of droplet actuation.

\section{Acknowledgements}

Vivek Ramadoss made valuable contributions to this work through the initial studies that he performed. These served as a foundation for the present paper. Nathan Crane would like to acknowledge support from NACE International, the University of South Florida Research Education Initiative Program under Grant Number FMMD04, and from NSF under CMMI-0927637 grant. Alex Volinsky would like to acknowledge support from the National Science Foundation under CMMI-0600266 grant. The authors would like to thank Hysitron, Inc. for providing blank tips and useful discussion. 
TABLE 1 Surface Evolver Model Parameters

\begin{tabular}{|l|l|}
\hline Variable & Value \\
\hline Gap width $(g)$ & $0.5 \mathrm{~mm}$ \\
\hline Surface Energy $\left(\gamma_{v}\right)$ & $0.072 \mathrm{~J} / \mathrm{m}^{2}$ \\
\hline $\begin{array}{l}\text { Liquid/Substrate Contact Angle } \\
\left(\theta_{o}\right)\end{array}$ & $110^{\circ}$ \\
\hline $\begin{array}{l}\text { Liquid/Measurement Plate } \\
\text { Contact Angle }\end{array}$ & $20^{\circ}$ \\
\hline \begin{tabular}{l} 
Plate side length $(L)$ \\
\hline Dielectric constant $\left(\varepsilon_{r}\right)$
\end{tabular} & $9 \mathrm{~mm}$ \\
\hline Dielectric thickness $(\delta)$ & 2.1 \\
\hline Voltage $(V)$ & $2.1 \mu \mathrm{m}$ \\
\hline Liquid Volume $\left(V_{f}\right)$ & $100 \mathrm{~V}$ \\
\hline Plate to Substrate Gap $(h)$ & $40.5 \mu \mathrm{l}$ \\
\hline
\end{tabular}

Figure 1 (Color online) Illustration of basic electrowetting concept and the equivalent lumped parameter circuit model.

Figure 2 (Color online) Illustration of the electrowetting force measurement method. a) Schematic representation of the measurement setup. b) Photos of the glass plate attached to the force transducer before and after contacting the liquid drop. c) Comparison of substrate setup and lumped-parameter circuit models for two common electrowetting force test modes.

Figure 3 (Color online) Illustration of basic Surface Evolver model geometry. 
Figure 4 (Color online) Analytical and Surface Evolver predictions of the electrowetting force as a function of displacement. The accuracy of the simple model decreases as the plate/substrate gap (h) decreases.

Figure 5 (Color online) Variation of lateral force and normal forces with rotational misalignment errors about each axis as predicted by Surface Evolver models. Lateral forces are relatively insensitive to alignment errors.

Figure 6 (Color online) Variation of normal and lateral forces with liquid volume as predicted by Surface Evolver models. Larger gaps show less sensitivity to changes in the liquid volume in both lateral and normal forces. Normal forces are much more sensitive to liquid volume than are lateral forces.

Figure 7 (Color online) Surface Evolver model results comparing lateral forces with liquid volume for constant plate/substrate gap and a constant liquid volume/projected volume ratio. The constant ratio produces more stable force measurements.

Figure 8 (Color online) Comparison of measured peak lateral forces with analytical and numerical predictions.

Figure 9 (Color online) EWF Lateral Force data for $1 \mathrm{mM} \mathrm{Na}_{2} \mathrm{SO}_{4}$ solution. a) Response to a series of DC voltage pulses. b) Plot of peak data for the $110 \mathrm{~V}$ pulse.

Figure 10 (Color Online) (a) Lateral force response to a step from 0 to $75 \mathrm{~V}$ shows some overshoot followed by a decaying oscillation. (b) Frequency response data for EWF lateral forces for three different liquids. 


\section{References}

1. F. Mugele and J. C. Baret, J. Phys. -Condes. Matter 17, R705 (2005).

2. D. Chatterjee, B. Hetayothin, A. R. Wheeler, D. J. King and R. L. Garrell, Lab Chip 6, 199 (2006).

3. S. Millefiorini, A. H. Tkaczyk, R. Sedev, J. Efthimiadis and J. Ralston, J. Am. Chem. Soc. 128, 3098 (2006).

4. P. Paik, V. K. Pamula, M. G. Pollack and R. B. Fair, Lab Chip 3, 28 (2003).

5. S. K. Cho, H. J. Moon and C. J. Kim, J Microelectromech Syst 12, 70 (2003).

6. Yuejun Zhao and Sung Kwon Cho, Lab on a Chip 7, 273 (2007).

7. P. Y. Chiou, H. Moon, H. Toshiyoshi, C. J. Kim and M. C. Wu, Sens. Actuator A-Phys. 104, 222 (2003).

8. K. Mohseni and E. S. Baird, Nanoscale Microscale Thermophys. Eng. 11, 99 (2007).

9. S. Kuiper and B. H. W. Hendriks, Appl. Phys. Lett. 85, 1128 (2004).

10. L. Dong, A. K. Agarwal, D. J. Beebe and H. R. Jiang, Nature 442, 551 (2006).

11. R. A. Hayes and B. J. Feenstra, Nature 425, 383 (2003).

12. C. G. Cooney, C. Y. Chen, M. R. Emerling, A. Nadim and J. D. Sterling, Microfluid. Nanofluid. 2, 435 (2006). 
13. H. J. J. Verheijen and M. W. J. Prins, Langmuir 15, 6616 (1999).

14. M. K. Kilaru, J. Heikenfeld, G. Lin and J. E. Mark, Appl. Phys. Lett. 90, 212906 (2007).

15. S. K. Fan, H. P. Yang, T. T. Wang and W. Hsu, Lab on a Chip 7, 1330 (2007).

16. A. Quinn, R. Sedev and J. Ralston, J Phys Chem B 109, 6268 (2005).

17. K. L. Wang and T. B. Jones, Appl. Phys. Lett. 86, 054104 (2005).

18. K. H. Kang, Langmuir 18, 10318 (2002).

19. T. B. Jones, Mech. Res. Commun. 36, 2 (2009).

20. H. J. J. 1. Verheijen and M. W. J. 1. Prins, Rev. Sci. Instrum. 70, 3668 (1999).

21. Sung Hee Ko, Horim Lee and Kwan Hyoung Kang, Langmuir 24, 1094 (2008).

22. Ilju Moon and Joonwon Kim, Sens Actuators A Phys 130-131, 537 (2006).

23. Abhay Vasudev and Zhe Jiang, 6 (2008).

24. Antonis I. Drygiannakis, Athanasios G. Papathanasiou and Andreas G. Boudouvis, Langmuir 25, 147 (2009).

25. E. Baird, P. Young and Kamran Mohseni, Microfluidics and Nanofluidics 3, 635 (2007).

26. E. S. Baird and K. Mohseni, Nanoscale Microscale Thermophys. Eng. 11, 109 (2007).

27. A. Kutana and K. P. Giapis, Nano Letters 6, 656 (2006). 
28. Li Guan, Guicun Qi, Sheng Liu, Hui Zhang, Zhong Zhang, Yanlian Yang and Chen Wang, Journal of Physical Chemistry C 113, 661 (2009).

29. J. Y. Chen, A. Kutana, C. P. Collier and K. P. Giapis, Science 310, 1480 (2005).

30. N. B. Crane, A. A. Volinsky, V. Ramadoss, M. Nellis, P. Mishra and Xiaolu Pang, Materia. Res. Soc. Symp. Proc. Volume 1052 DD8.1 (2007).

31. K. Brakke, Exp. Math 1, 141 (1992).

32. J. Lienemann, A. Greiner and J. G. Korvink, IEEE Trans. Comput-Aided Des. Integr. Circuits Syst. $25,234(2006)$.

33. V. Ramadoss and N. B. Crane, 2008 ASME International Mechanical Engineering Congress IMECE2008-68023 (2008).

34. M. Pendergast, A. A. Volinsky, X. Pang and R. Shields, Mat. Res. Soc. Symp. Proc. Volume 1085E 1085E, T5.10 (2008).

35. B. Raj, M. Dhindsa, N. R. Smith, R. Laughlin and J. Heikenfeld, Langmuir 25, 12387 (2009). 